\title{
Diabetes Is Associated with Increased Rate of Cognitive Decline in Questionably Demented Elderly
}

\author{
Ramit Ravona-Springer $^{\mathrm{a}}$ Xiaodong Luo $^{\mathrm{b}}$ James Schmeidler ${ }^{\mathrm{b}}$ \\ Michael Wysockib ${ }^{b}$ Gerson Lesser ${ }^{c}$ Michael Rapp ${ }^{d}$ Karen Dahlman ${ }^{b}$ \\ Hillel Grossman ${ }^{b}$ Vahram Haroutunian ${ }^{b}$ Michal Schnaider Beeri ${ }^{b}$ \\ ${ }^{a}$ Memory Clinic, Sheba Medical Center, Ramat Gan, Israel; ${ }^{b}$ Department of Psychiatry, Mount Sinai School of \\ Medicine, and ${ }^{\mathrm{C} J e w i s h ~ H o m e ~ a n d ~ H o s p i t a l, ~ N e w ~ Y o r k, ~ N . Y ., ~ U S A ; ~}{ }^{\mathrm{d}}$ Department of Psychiatry, Campus Charité Mitte, \\ Humboldt University, Berlin, Germany
}

Key Words

Dementia comorbidity $\cdot$ Cognitive decline $\cdot$ Diabetes

\begin{abstract}
Background: This study examines whether the association of diabetes with the rate of cognitive decline varies according to dementia severity. Methods: Longitudinal study on subjects residing in nursing homes and assisted living $(\mathrm{n}=$ 342). The Mini Mental State Examination (MMSE) was used to measure the rate of cognitive decline in diabetic and nondiabetic subjects who were nondemented (Clinical Dementia Rating, $C D R=0 ; n=125)$, questionably demented $(C D R=0.5$; $n=58)$ or frankly demented (CDR $\geq 1 ; n=89)$ at baseline. Diagnosis of diabetes was ascertained by review of medical records and history. Results: Diabetes was associated with an increased rate of decline in the MMSE score of questionably demented subjects $(p<0.0001)$. In frankly demented subjects, diabetes tended to be associated with less cognitive decline $(p=0.04)$. Diabetes was not associated with the rate of MMSE decline in nondemented subjects $(p=0.89)$. Conclusion: In individuals with questionable dementia (CDR = 0.5 ), diabetes is associated with a faster rate of cognitive decline as measured by the MMSE, but not in nondemented $(C D R=0)$ or frankly demented $(C D R \geq 1)$ individuals.
\end{abstract}

Copyright $\odot 2010$ S. Karger AG, Basel

\section{KARGER}

Fax +4161306 1234 E-Mail karger@karger.ch www.karger.com (c) 2010 S. Karger AG, Basel

Accessible online at: www.karger.com/dem

\section{Introduction}

The prevalence of both diabetes and dementia increases with age, reaching rates of up to $14 \%$ for dementia [1] and approximately $14 \%$ for diabetes at the of age of 80 and above [2]. Type II diabetes has been demonstrated by many [3-8], but not all $[9,10]$ studies to be a risk factor for cognitive decline, mild cognitive impairment and dementia [3], both vascular dementia [11, 12] and Alzheimer's disease [13]. The deleterious effects of diabetes on cognition may not only be evident in higher rates of conversion to dementia, but also in faster cognitive deterioration than that typically found in those without diabetes. Previous studies describe varied findings regarding effects of diabetes on the rate of cognitive decline with the majority suggesting an increased rate of decline [14] but some suggesting no association [14]. Variations may be attributed to differences in operationally different definitions for diabetes, duration of exposure to diabetes, antidiabetic treatments, degree of glycemic control, duration of cognitive follow-up, cognitive status at baseline and cognitive assessment tools.

Interestingly, despite diabetes being a potentially modifiable risk factor and despite the relative consistency in reports demonstrating the association of diabetes with

Ramit Ravona-Springer

Memory Clinic, Sheba Medical Center

Ramat Gan 52621 (Israel)

Tel. +972 54255 0676, Fax +97235346628

E-Mail ramit.ravona@sheba.health.gov.il 
cognitive compromise, cognitive assessment is usually not performed as part of the routine evaluation and follow-up of diabetic patients. If such assessments were to be made standard, the selection of optimal screening tools for detection of cognitive changes in diabetic patients as part of routine clinical assessment would likely vary according to a patient's age and cognitive status. The aim of the current study was to assess the effect of diabetes on the rate of $\operatorname{cog}$ nitive decline using the Mini Mental State Examination (MMSE) [15], in a cohort of elderly subjects with a broad range of dementia severity. Subjects were stratified according to the Clinical Dementia Rating (CDR) scale to nondemented $(\mathrm{CDR}=0)$, questionably demented $(\mathrm{CDR}=$ $0.5)$ and demented $(\mathrm{CDR} \geq 1)$ groups, thus allowing for examination of the effect of diabetes on the MMSE score decline at different stages of the dementia course.

The cohort consists of nursing homes and assisted living residents, who constitute a growing proportion of the elderly population, reaching up to $40 \%$ of Americans over the age of 65 years [16]. Thus, characterizing the relationship of diabetes with the course of cognitive decline in this segment of the population is warranted.

\section{Methods}

\section{Subjects}

The sample consisted of 342 residents of the Jewish Home and Hospital (JHH) Bronx and Manhattan divisions, both skilled nursing facilities, and Kittay House, an assisted living facility affiliated with the JHH. The Mount Sinai School of Medicine has had an academic affiliation with the JHH for more than 25 years. As part of a program project on Clinical and Biological Studies of early Alzheimer's disease, each new long-term resident admitted to the JHH is administered an MMSE and assessed for further inclusion in memory and aging projects led by the Department of Psychiatry, Mount Sinai School of Medicine. All participating subjects were English or Spanish speakers (staff included both English- and Spanish-speaking psychometricians, and the psychometric instruments were administered in the subject's language of choice). Subjects were included in the study if: their baseline MMSE score was between 10 and 30; they had at least 1 follow-up assessment; their medical records were available for review of diabetes status, and they had complete demographic data (age, gender, race and education). Subjects were not included if they had a baseline diagnosis of cerebrovascular disease or a neurological or psychiatric disorder that might affect cognition, such as Parkinson's disease or schizophrenia. The mean length of follow-up time was 2.95 years $(\mathrm{SD}=1.67)$. The total number of assessments among the 342 subjects was 1,234, and the range of number of annual assessments per subject was $2-16($ mean $=3.61 ; \mathrm{SD}=2.64)$. Subjects with baseline MMSE scores below 10 were excluded in order to minimize floor effects. All follow-up assessments after the subject reached 0 points on the MMSE were also excluded.

Rate of Cognitive Decline in Diabetic Elderly
Assessment Procedures

Mini Mental State Examination

Each subject was serially assessed at approximately annual intervals with an MMSE (assessment intervals were within predefined windows of $12 \pm 3$ months to avoid confounds introduced by medical illness and other life events). The MMSE is a commonly used 30-point scale for assessing cognitive function in the following areas: orientation, registration, attention and calculation, recall, language and praxis. MMSE administration was performed according to existing standards [15], with the spelling of the word 'world' backwards used exclusively for the 'attention and calculation' domain, as opposed to the alternative serial 7s.

Clinical Dementia Rating

A CDR scale was administered to each participant at MMSE baseline. The CDR is an established instrument for assessment of cognitive function and performance on a 5 -point scale in both clinical and research settings in 6 domains: memory, orientation, judgment and problem solving, community affairs, home and hobbies, and personal care [17]. A score for each domain is established by semistructured interviews with the participant, as well as a separate informant interview with an individual familiar with the participant (usually a family member, nurse or certified nursing assistant). Scores are 0 (nondemented), 0.5 (questionable dementia), 1 (mild dementia), 2 (moderate dementia) and 3 (severe dementia). All psychometricians were certified in the CDR administration (http://www.alz.washington.edu/NONMEMBER/cdr2.html).

For the purposes of the present analysis, subjects were stratified by dementia severity as nondemented (CDR $=0)$, questionably demented $(\mathrm{CDR}=0.5)$ or demented $(\mathrm{CDR} \geq 1)$, and the MMSE (not the CDR) was the dependent variable. Loss of independence in subjects included in the $\mathrm{CDR}=0$ group was due to reasons other than cognitive, usually sensory limitations and physical disabilities.

The diagnosis of mild cognitive impairment requires neuropsychological assessment [18] (beyond the MMSE) that was not available in this study. Although the $\mathrm{CDR}=0.5$ category and mild cognitive impairment diagnosis are correlated, there are discrepancies in their classifications [19]. Therefore, we designate the $\mathrm{CDR}=0.5$ group as 'questionably demented' rather than calling them 'with mild cognitive impairment'.

\section{Diabetes Status}

The JHH maintains extensive medical records on all residents, with a medical history at admission, a medical examination monthly thereafter and a fasting blood glucose level at least yearly. Medical management, including medication administration, is primarily performed by staff. Patients were diagnosed with diabetes by a geriatrician or an internist based on the American Diabetes Association criteria (symptoms of diabetes plus casual plasma glucose concentration $\geq 200 \mathrm{mg} / \mathrm{dl}$; fasting plasma glucose $\geq 126 \mathrm{mg} / \mathrm{dl}$; 2-hour plasma glucose $\geq 200 \mathrm{mg} / \mathrm{dl}$ during an oral glucose tolerance test). Impaired fasting glucose was defined as blood level of 110-125 mg/dl (6.1-7.0 mmol/l). Subjects taking antidiabetic medications also received a diagnosis of diabetes. The diagnosis was ascertained from detailed review of all medical records and medical history. Subjects who had high fasting blood glucose levels but did not match clinical criteria for diabetes were not included in the analysis. The present analysis included only subjects with type 2 diabetes or no record of diabetes (absence of 
Table 1. Descriptive analysis by CDR group

\begin{tabular}{|c|c|c|c|c|c|c|c|c|c|c|c|c|c|}
\hline \multirow[b]{2}{*}{ Diabetic status: } & \multicolumn{3}{|c|}{$\mathrm{CDR}=0$} & \multicolumn{3}{|c|}{$\mathrm{CDR}=0.5$} & \multicolumn{3}{|c|}{$\mathrm{CDR} \geq 1$} & \multicolumn{3}{|c|}{ Total by diabetic status } & \multirow[t]{2}{*}{ Total } \\
\hline & $\mathrm{D}$ & ND & $\mathrm{p}$ & $\mathrm{D}$ & ND & $\mathrm{p}$ & $\mathrm{D}$ & ND & $\mathrm{p}$ & $\mathrm{D}$ & ND & $\mathrm{p}$ & \\
\hline Number & 18 & 177 & & 13 & 45 & & 20 & 69 & & 51 & 291 & & 342 \\
\hline Mean baseline age, years & $\begin{array}{c}78.31 \\
(10.72)\end{array}$ & $\begin{array}{r}85.83 \\
(6.94) \\
\end{array}$ & 0.009 & $\begin{array}{c}84.70 \\
(7.72) \\
\end{array}$ & $\begin{array}{r}87.97 \\
(6.17) \\
\end{array}$ & 0.18 & $\begin{array}{c}83.18 \\
(7.18)\end{array}$ & $\begin{array}{c}86.81 \\
(6.58) \\
\end{array}$ & 0.051 & $\begin{array}{r}81.85 \\
(8.96) \\
\end{array}$ & $\begin{array}{r}86.39 \\
(6.77) \\
\end{array}$ & 0.001 & $\begin{array}{r}85.71 \\
(7.30)\end{array}$ \\
\hline Mean education, years & $\begin{array}{r}13.67 \\
(2.85) \\
\end{array}$ & $\begin{array}{c}14.74 \\
(3.14)\end{array}$ & 0.15 & $\begin{array}{r}11.15 \\
(3.21)\end{array}$ & $\begin{array}{r}11.09 \\
(3.71)\end{array}$ & 0.95 & $\begin{array}{c}11.70 \\
(2.81)\end{array}$ & $\begin{array}{r}11.75 \\
(3.70)\end{array}$ & 0.94 & $\begin{array}{l}12.26 \\
(3.06)\end{array}$ & $\begin{array}{r}13.47 \\
(7.72)\end{array}$ & 0.01 & $\begin{array}{r}13.29 \\
(3.65)\end{array}$ \\
\hline Mean baseline MMSE & $\begin{array}{l}26.56 \\
(2.53)\end{array}$ & $\begin{array}{r}28.03 \\
(1.74)\end{array}$ & 0.03 & $\begin{array}{l}24.08 \\
(2.63)\end{array}$ & $\begin{array}{c}23.11 \\
(2.64)\end{array}$ & 0.26 & $\begin{array}{l}18.90 \\
(4.15)\end{array}$ & $\begin{array}{r}18.80 \\
(2.89)\end{array}$ & 0.91 & $\begin{array}{c}22.92 \\
(4.69)\end{array}$ & $\begin{array}{l}25.08 \\
(4.49)\end{array}$ & 0.003 & $\begin{array}{r}24.76 \\
(4.58)\end{array}$ \\
\hline Females, \% & 38.9 & 71.2 & 0.005 & 92.3 & 77.8 & 0.2 & 85 & 82.6 & 0.5 & 70.6 & 74.9 & 0.3 & 74.3 \\
\hline Caucasians, \% & 88.9 & 93.8 & 0.4 & 72.9 & 88.9 & 0.2 & 60 & 82.6 & 0.04 & 74.5 & 90.4 & 0.003 & 88.0 \\
\hline
\end{tabular}

$\mathrm{D}=$ Diabetic $; \mathrm{ND}=$ nondiabetic. Figures in parentheses indicate standard deviations.

reported history or medications for diabetes, and failure to meet blood chemistry-based criteria). Medication use statuses were not consistently available to us, nor were measures of diabetes duration prior to $\mathrm{JHH}$ admission.

\section{Covariates}

Age, gender (male reference group), race (Caucasian reference group) and years of education (continuous for descriptive purposes and trichotomized to $<8,8-12$ and $>12$ for the generalized estimating equation, GEE, analysis described below, with $>12$ years of education the reference group) were used as covariates due to their known associations with cognitive performance and impairment [20, 21]. The covariates, and baseline MMSE, are summarized in table 1, by diabetic status and CDR status.

GEE were used to model the MMSE scores over time within each CDR group. GEE is a generalization of the linear regression models that also accounts for within-subject correlation among the MMSE scores for a person over time. GEE provide consistent estimators of both the regression parameters and their corresponding variances, which are necessary for valid statistical inference. When fitting the GEE, we specified an arbitrary within-subject correlation matrix to obtain consistent estimators of the regression parameters, and then used the residuals based on the provided estimators to calculate the variances of the estimators.

\section{Statistical Analysis}

This study included all available data from an ongoing longitudinal study; so, the starting point of each subject was the entry into the longitudinal study, and the 'end point' was the last observation within the relevant CDR category, as described below.

We analyzed the baseline covariates overall and by diabetes status and CDR status. For continuous covariates such as baseline age and MMSE, we used 2-sample t tests to compare the means of the diabetic and nondiabetic groups. We assumed unequal variances and used Satterthwaite's method to provide approximate degrees of freedom. For categorical covariates such as gender, race and level of education, we used the Pearson $\chi^{2}$ test to compare the differences between diabetic and nondiabetic subjects.
The GEE models had the MMSE scores at successive follow-up time points as dependent variables and diabetes status, the followup time from baseline and the interaction between the diabetic status and time as independent variables, controlling for MMSE at baseline, age at baseline, gender, race and education. Positive diabetes diagnosis was the reference group for diabetes status. Thus, a significant result for the coefficient of the follow-up time would signify a significant change over time in MMSE in the diabetes-positive group. Likewise, a significant result for the coefficient of the interaction between the follow-time and the diabetic status would signify a significant difference in rate of MMSE decline in the diabetes-negative group compared with the diabetespositive group. SAS GENMOD (procedure for the generalized linear models) was used to fit the GEE. For each group, a figure presents fitted models for change in MMSE over time for subjects with and without diabetes, with the covariates evaluated at the mean levels obtained from the overall sample.

Since the objective of the study was to examine the relationship of diabetes with cognitive decline within each stage of dementia course, primary analysis was limited to the first CDR category in which the subject had at least 2 observations. No distinction was made between subjects who had additional observations in other CDR categories, subjects who dropped out within a CDR category and subjects who are continuing to participate in the ongoing longitudinal study and have not yet changed to a higher CDR. Mixed regression models were used to address the discrepant spacing of observations in different individuals within the same CDR category.

For the primary analyses, a separate GEE was fitted for each of the CDR groups. MMSE observations for a subject were included in an analysis until a later CDR score differed from the baseline CDR score. Nonetheless, in the event of a sequence of 5 CDR observations, for which the first 2 and last 2 fell into the same CDR group, but the middle CDR score differed, all 5 MMSE values were included in the analysis. The consistency of the similar CDR observations was sufficient to disregard the apparent exception in the middle. 
Table 2. Covariate associations

\begin{tabular}{|c|c|c|c|c|c|c|c|c|c|}
\hline & \multicolumn{3}{|c|}{$\mathrm{CDR}=0$} & \multicolumn{3}{|c|}{$\mathrm{CDR}=0.5$} & \multicolumn{3}{|c|}{$\mathrm{CDR} \geq 1$} \\
\hline & $\mathrm{p}$ & est. & SE & $\mathrm{p}$ & est. & SE & $\mathrm{p}$ & est. & SE \\
\hline Age & 0.12 & -0.03 & 0.02 & 0.20 & 0.08 & 0.06 & 0.79 & -0.03 & 0.10 \\
\hline Gender & 0.89 & 0.04 & 0.31 & 0.56 & 0.59 & 1.02 & 0.37 & 1.25 & 1.39 \\
\hline MMSE at baseline & $<0.0001$ & 0.45 & 0.07 & 0.08 & 0.20 & 0.11 & $<0.0001$ & 0.67 & 0.15 \\
\hline Education $<8$ years & 0.20 & -0.98 & 0.76 & 0.03 & -2.18 & 1.00 & 0.40 & 1.43 & 1.67 \\
\hline Education $8-12$ years & 0.92 & 0.03 & 0.27 & 0.03 & -1.87 & 0.84 & 0.05 & 2.47 & 1.29 \\
\hline Race & 0.85 & -0.07 & 0.37 & 0.90 & -0.14 & 1.04 & 0.09 & 2.53 & 1.50 \\
\hline Diabetes status & 0.47 & 0.37 & 0.52 & $<0.0001$ & -4.61 & 0.89 & 0.04 & 3.99 & 1.99 \\
\hline Time & 0.78 & -0.05 & 0.18 & 0.0001 & -1.53 & 0.39 & 0.01 & -1.49 & 0.61 \\
\hline Diabetes/time interaction & 0.89 & -0.03 & 0.20 & $<0.0001$ & 2.29 & 0.50 & 0.04 & -1.43 & 0.70 \\
\hline
\end{tabular}

Reference groups are as follows: gender - male; education - education $>12$ years; race - white; diabetes status - positive diabetes diagnosis. est. $=$ Estimated; $\mathrm{SE}=$ standard error.

In secondary analyses, instead of restricting the analysis to each subject's initial CDR group, a subject who moved from one CDR group to another at the follow-up could contribute data for analysis of more than one CDR group, as if he or she were a different subject in each group. If a subject had more than 1 sequence of at least 2 observations in the same CDR group, only the longest was selected for analysis. If they were of equal length, the first following a lower CDR group was selected. This increased the numbers of subjects in the questionably demented (from 58 to 64) and demented groups (from 89 to 107).

\section{Results}

As presented in table 1, of the 342 subjects, 51 (14.9\%; mean age $=86.4$ years, mean years of education $=13.5$ ) had a diagnosis of diabetes, and 291 (85.1\%; mean age = 81.8 years, mean years of education $=12.2$ ) did not have a diagnosis of diabetes. The mean age of the entire sample was 85.7 years $(\mathrm{SD}=7.3)$ at baseline, and the mean education level was 13.3 years $(\mathrm{SD}=3.65)$. For the entire sample, subjects with a diagnosis of diabetes were younger $(\mathrm{p}=0.001)$, less educated $(\mathrm{p}=0.01)$, with a lower baseline MMSE score $(\mathrm{p}=0.003)$ and with a lower percentage of Caucasians $(\mathrm{p}=0.003)$.

The sample was stratified by CDR score at baseline. One hundred ninety-five subjects were nondemented at baseline ( $\mathrm{CDR}=0), 58$ subjects were questionably demented $(C D R=0.5)$, and 89 were mildly to severely demented $(\mathrm{CDR} \geq 1)$. In separate comparisons by diabetes status for the three CDR score groups, there were no significant differences in the level of education. Diabetic subjects in the CDR $=0$ group were younger $(p=0.009$, $\mathrm{t}=2.91$, d.f. $=18.5)$, included fewer female subjects $(\mathrm{p}=$ $0.005, \chi^{2}=7.86$, d.f. $=1$ ) and had a lower MMSE score at baseline $(\mathrm{p}=0.03)$. In the $\mathrm{CDR}=0.5$ group, diabetic subjects did not differ significantly from nondiabetic subjects in age, education, baseline MMSE, gender or race. The CDR $\geq 1$ diabetic subjects tended to be younger $(\mathrm{p}=0.051)$ and included fewer Caucasian subjects $(\mathrm{p}=$ $0.04)$.

Table 2 presents the parameter estimates of the included covariates (age, gender, education, race) and the independent variables (diabetic status, time and diabetes by time) for each of the three groups. In the nondemented $(\mathrm{CDR}=0)$ group, there was no significant time effect or interaction of time with diabetes status. The only significant predictor of MMSE decline was baseline MMSE $(p<0.0001)$. The lack of significant changes over time or interaction of diabetes status with time indicates that individual differences in baseline MMSE were maintained throughout the period in which participants were nondemented.

In the questionably demented $(\mathrm{CDR}=0.5)$ group, there were a strong time effect $(\mathrm{p}=0.0001)$ and diabetes by time interaction $(\mathrm{p}<0.0001)$ with diabetic subjects declining by 2.3 more MMSE points per year compared to nondiabetic subjects. Education also had a significant relationship with baseline MMSE in the questionably demented group, with lower MMSE scores for subjects who had not attended high school or had not gone beyond high school (each $\mathrm{p}=0.03$ ) compared to subjects with more education. The association between baseline MMSE and rate of MMSE decline approached significance $(\mathrm{p}=$ 0.008 ) in this group. 
In the demented $(\mathrm{CDR} \geq 1)$ group, there were a time effect $(p=0.01)$ and diabetes by time interaction with nondiabetics declining at a faster rate (1.4 points per year; $\mathrm{p}=0.04)$ than diabetic subjects. Baseline MMSE was predictive of MMSE decline $(\mathrm{p}<0.0001)$.

In secondary analyses, in which a single subject could contribute data for analysis in as many as three CDR categories, the diabetes by time interaction results remained essentially identical for the $\mathrm{CDR}=0.5$ group. Diabetic subjects in the $\mathrm{CDR}=0.5$ group declined significantly faster in MMSE scores compared to nondiabetic subjects (estimate $=2.03 ; \mathrm{p}<0.0001, \mathrm{Z}=4.70$ ). Diabetic subjects in the CDR $\geq 1$ group did not differ in their rates of decline when compared to nondiabetic subjects (estimate = $-0.49 ; \mathrm{p}=0.4, \mathrm{Z}=-0.84)$.

\section{Discussion}

For diabetic subjects with questionable dementia at baseline, the rate of cognitive decline as measured by the MMSE was significantly faster relative to nondiabetic questionably demented subjects (2.3 more MMSE points per year). In contrast, for demented subjects, nondiabetics declined slightly faster than diabetics in one analysis (1.4 more MMSE points per year). Decline in MMSE score was not observed in diabetic as well as nondiabetic subjects with no dementia. Results did not change for the questionably demented subjects when using an alternative analysis with a larger sample size. For demented subjects, the results for the larger sample did not show a significant association of diabetes with the rate of cognitive decline.

These results suggest that diabetic elderly individuals in predementia stages may be at higher risk for further cognitive decline compared to nondiabetic individuals. Since there are no differences in cognitive decline in the nondemented subjects between diabetics and nondiabetics, the results may suggest that the contribution of diabetes in this group is not profound enough to affect clinically evident cognitive decline, but may rather be an exacerbating condition once cognitive deterioration has already begun. It is also possible that the MMSE is not sensitive enough to detect subtle effects of diabetes on cognition in subjects with $\mathrm{CDR}=0$, as suggested by the very low variation among subjects' slopes. Lack of variation in the rate of MMSE deterioration in this group can be explained by sufficient cognitive reserve [22] to compensate for the potential detrimental impact of diabetes. Of note, however, is that cognitive stability was found in other longitudinal studies of cognitively intact elderly in- dividuals even when cognitive function was also assessed by a broader neuropsychological battery $[23,24]$.

In contrast, both the $\mathrm{CDR}=0.5$ and $\mathrm{CDR} \geq 1$ groups had substantial variation among subjects' slopes, permitting detection of deleterious effects of diabetes if present. No significant interaction of diabetes with MMSE change over time was found consistently in the $\mathrm{CDR} \geq 1$ group; perhaps in individuals with frank dementia, the brain might be sufficiently compromised by neuropathology so that any additional impact of diabetes becomes inconsequential to cognitive function, at least as measured by the MMSE.

It is also important to note that in the diabetes group, MMSE deterioration occurred within the same CDR category $(\mathrm{CDR}=0.5)$. Change of CDR category from 0.5 to $\geq 1$ reflects decline in functional status. Decline in MMSE score in the absence of functional decline may represent a transitional phase in the course of cognitive deterioration, during which the efficacy of strategies introduced earlier in the course of diabetes - before any observed cognitive deterioration - may be most measurable. Alternatively, this may indicate that there is an optimal stage for the introduction of dementia prevention strategies in diabetic subjects, that is when the earliest signs of cognitive change occur but before functional decline sets in. This would underscore the importance of routine cognitive assessment in elderly diabetic subjects.

In all CDR categories, age did not affect the rate of MMSE deterioration. This finding is consistent with reports from several other studies [25-29] and suggests that the role of age as the most important risk factor for dementia [30] may not necessarily overlap with its role in disease progression.

Previous studies assessing the association of diabetes with the rate of cognitive decline have reported conflicting results. Some studies have reported that diabetes affects the rate of cognitive decline (as measured by the MMSE), though effect sizes varied. In two large-scale, population-based studies, a higher rate of cognitive decline was detected in diabetic versus nondiabetic subjects using the MMSE; however, the MMSE had limited sensitivity relative to other tests (Digit Symbol Test or Trail Making Test) [31, 32]. Similarly, in a 4-year follow-up study of subjects with normal cognition at baseline (MMSE >26), diabetic subjects were likely to decline on domain-specific tests such as the Auditory Verbal Learning Test (assessing memory), Finger Tapping Test (assessing psychomotor speed), Digit Symbol Substitution and Test of Facial Recognition (assessing attention) but not on the MMSE [1]. Differences in the extent of effects of dia- 
betes on different tools for cognitive assessment may be attributed to lower sensitivity of the MMSE (or modified MMSE) compared to more detailed or taxing measures of cognitive function and possible ceiling effects; however, it should be taken into consideration that in the above-mentioned studies, subjects were not stratified according to baseline cognitive status. Thus, as suggested by our results, effects of diabetes on cognition, and on MMSE in particular, might have been masked in these other studies by a combination of different dementia levels in subject groups at baseline.

Other studies have also reported no association between the rate of MMSE score deterioration and diabetes status [33-35]. Such results may be attributed to inclusion of subjects with a wide age range at baseline (42-89 years) [33], an overtly restrictive definition of cognitive decline (i.e. $\geq 2$ MMSE points per year) on an instrument with limited sensitivity [34] and a lack of stratification using functional and global scales such as the CDR [35].

Domain-specific neuropsychological tests may be more sensitive than the MMSE in certain subject populations. However, diabetes has been hypothesized to contribute to cognitive decline through several mechanisms [36-39] and by more than one brain pathology (vascular pathology as well as atrophy) [40]. Different pathologies may be associated with different patterns of decline in specific cognitive domains [40]. Under these circumstances, a multi-domain test such as the MMSE, which assesses orientation, registration, attention and calculation, recall, language and praxis [15], may be effective enough for use as a screening tool; the current study demonstrates that the MMSE has adequate sensitivity to be used in patients with questionable or early-stage cognitive impairment $(\mathrm{CDR}=0.5)$.

The main limitation of our study is the lack of more detailed information on diabetes, such as duration of disease, glycemic control, long-term antidiabetic medication use, weight and diet, factors that may be mediators of the relationships between diabetes and cognitive decline. This is particularly relevant with regard to medication use, which has been associated with $\mathrm{AD}$ neuropathology [41]. Measurement of the effect of antidiabetic medication and other diabetes characteristics and complications on clinically assessed cognition requires application of methodologies different than the one used in our study and much larger patient populations. An additional limitation was that while the effect of macrovascular pathology was partly abated by the exclusion of patients with cerebrovascular disease at baseline, the potential contribution of incident macrovascular, as well as microvascular pathology to cognitive decline was not measured in this study.

This study characterizes the effect of diabetes on cognitive decline in persons constituting a large and rapidly growing segment of the elderly population [16]. Nursing home and assisted-living residents have been reported to differ from community-residing elderly individuals in some measures of morbidity and mortality [42-44]. This is particularly relevant to medication use and control of disease, since the direct care settings provide strict medication and laboratory follow-up management to all residents, irrespective of their cognitive status. However, the differences in level of care, mortality and morbidity also raise the possibility that the results reported here may not be entirely applicable to community-residing elderly individuals.

Our study demonstrates that a simple, easily administered screening tool has adequate sensitivity to monitor cognitive changes in diabetic elderly subjects with subtle or early-stage cognitive impairment. This tool can be used as part of a routine follow-up in such populations, enabling assessment of feasibility and cost-effectiveness of antidiabetic treatments in dementia prevention or amelioration. Implementation of strategies for routine follow-up of other diabetes-related complications has enabled the assessment of the risk-benefit ratio of antidiabetes treatment in their prevention or amelioration [45]. Interestingly, despite potential advantages, cognitive assessment is still not recommended routinely in diabetic patients $[45,46]$.

Possible implications of our findings include: (1) in questionably demented individuals who suffer from diabetes, the MMSE, an easily administered cognitive screening tool, should be considered for routine followup of cognitive status; (2) in elderly diabetic subjects, the effects of treatments aimed to prevent, moderate or postpone dementia may most easily be measured (with a simple and easily administered tool) in questionably demented individuals, and (3) future studies should identify optimal tools for routine measurement of cognitive decline in nondemented and demented diabetic populations and their interactions with functional status.

\section{Acknowledgements}

This study was supported by NIA grants K01 AG023515-01 and RO1 AG034087, and the Graubard Fund for Dr. Beeri, P01 AG02219 and the Berkman Trust for Dr. Haroutunian, and P50 AG05138 for Dr. Sano. We thank Dr. Yoram Braw, PhD, EmotionCognition Research Center, Shalvata Mental Health Center, HodHasharon, Israel. 


\section{References}

$\checkmark 1$ Thomas VS, et al: Estimating the prevalence of dementia in elderly people: a comparison of the Canadian Study of Health and Aging and National Population Health Survey approaches. Int Psychogeriatr 2001;13(suppl 1):169-175.

$>2$ Wild S, et al: Global prevalence of diabetes: estimates for the year 2000 and projections for 2030. Diabetes Care 2004;27:1047-1053.

$\checkmark 3$ Schnaider Beeri M, et al: Diabetes mellitus in midlife and the risk of dementia three decades later. Neurology 2004;63:1902-1907.

$\checkmark 4$ Whitmer RA, et al: Midlife cardiovascular risk factors and risk of dementia in late life. Neurology 2005,64:277-281.

5 Janson J, et al: Increased risk of type 2 diabetes in Alzheimer disease. Diabetes 2004;53: 474-481.

6 Peila R, Rodriguez BL, Launer LJ: Type 2 diabetes, APOE gene, and the risk for dementia and related pathologies: the Honolulu-Asia Aging Study. Diabetes 2002;51:1256-1262.

$\checkmark 7$ Roberts RO, et al: Association of duration and severity of diabetes mellitus with mild cognitive impairment. Arch Neurol 2008;65: 1066-1073.

$>8$ Arvanitakis Z, et al: Diabetes mellitus and risk of Alzheimer disease and decline in cognitive function. Arch Neurol 2004;61:661666.

$\checkmark 9$ MacKnight $C$, et al: Diabetes mellitus and the risk of dementia, Alzheimer's disease and vascular cognitive impairment in the Canadian Study of Health and Aging. Dement Geriatr Cogn Disord 2002;14:77-83.

10 Hassing LB, et al: Diabetes mellitus is a risk factor for vascular dementia, but not for $\mathrm{Alz}$ heimer's disease: a population-based study of the oldest old. Int Psychogeriatr 2002;14: 239-248.

$\checkmark 11$ Raffaitin C, et al: Metabolic syndrome and risk for incident Alzheimer's disease or vascular dementia: the Three-City Study. Diabetes Care 2009;32:169-174.

12 Stewart R, Liolitsa D: Type 2 diabetes mellitus, cognitive impairment and dementia. Diabet Med 1999;16:93-112.

$13 \mathrm{Xu} \mathrm{W}$, et al: Mid- and late-life diabetes in relation to the risk of dementia: a populationbased twin study. Diabetes 2009;58:71-77.

- 14 Cukierman T, Gerstein HC, Williamson JD: Cognitive decline and dementia in diabetes - systematic overview of prospective observational studies. Diabetologia 2005;48: 2460-2469.
15 Folstein M, Folstein S: Mini-Mental State: a practical method for grading the cognitive state of patients for the clinician. J Psychiatr Res 1975;12:189-198.

16 Kemper P, Murtaugh CM: Lifetime use of nursing home care. N Engl J Med 1991;324: 595-600.

17 Morris J: The clinical dementia rating (CDR): current version and scoring rules. Neurology 1993;43:2412-2414.

18 Petersen RC, et al: Mild cognitive impairment: clinical characterization and outcome. Arch Neurol 1999;56:303-308.

19 Saxton J, et al: Functional and cognitive criteria produce different rates of MCI. J Neurol Neurosurg Psychiatry 2009;80:737-743.

20 Jones R, Gallo J: Education bias in the MiniMental State Examination. Int Psychogeriatr 2001;13:299-310.

21 Dufouil C, Alperovitch A, Tzourio C: Influence of education on the relationship between white matter lesions and cognition. Neurology 2003;60:831-836.

22 Stern Y: Cognitive reserve and Alzheimer disease. Alzheimer Dis Assoc Disord 2006; 20(3 suppl 2):S69-S74.

23 Rubin EH, et al: A prospective study of cognitive function and onset of dementia in cognitively healthy elders. Arch Neurol 1998;55: 395-401.

24 Galvin JE, et al: Predictors of preclinical Alz heimer disease and dementia: a clinicopathologic study. Arch Neurol 2005;62:758-765.

25 Doody RS, Massman P, Dunn JK: A method for estimating progression rates in Alzheimer disease. Arch Neurol 2001;58:449-54.

26 McCarten JR, et al: Patient age influences recognition of Alzheimer's disease. J Gerontol A Biol Sci Med Sci 2008;63:625-628.

$\checkmark 27$ Small BJ, Backman L: Predictors of longitudinal changes in memory, visuospatial, and verbal functioning in very old demented adults. Dement Geriatr Cogn Disord 1998;9: 258-266.

28 Small BJ, et al: Cognitive changes in very old persons with dementia: the influence of demographic, psychometric, and biological variables. J Clin Exp Neuropsychol 1997;19: 245-260.

29 Mielke MM, et al: Vascular factors predict rate of progression in Alzheimer disease. Neurology 2007;69:1850-1858.

30 Ott A, et al: Incidence and risk of dementia: the Rotterdam Study. Am J Epidemiol 1998; 147:574-580

-31 Haan MN, et al: The role of APOE epsilon4 in modulating effects of other risk factors for cognitive decline in elderly persons. JAMA 1999;282:40-46.
32 Gregg EK, et al: Is diabetes associated with cognitive impairment and cognitive decline among older women? Study of Osteoporotic Fractures Research Group. Arch Intern Med 2000;160:174-180.

33 Kanaya AM, et al: Change in cognitive function by glucose tolerance status in older adults: a 4-year prospective study of the Rancho Bernardo study cohort. Arch Intern Med 2004;164:1327-1333.

34 Kalmijn S, et al: Cardiovascular disease, the apolipoprotein e4 allele, and cognitive decline in a community-based study of elderly men. Stroke 1996;27:2230-2235.

35 van den Berg E, et al: The impact of diabetes mellitus on cognitive decline in the oldest of the old: a prospective population-based study. Diabetologia 2006;49:2015-2023.

-36 Farris W, et al: Insulin-degrading enzyme regulates the levels of insulin, amyloid betaprotein, and the beta-amyloid precursor protein intracellular domain in vivo. Proc Natl Acad Sci USA 2003;100:4162-4167.

$>37$ Qiu WQ, et al: Insulin-degrading enzyme regulates extracellular levels of amyloid beta-protein by degradation. J Biol Chem 1998;273:32730-32738

38 Smith MA, Sayre LM, Perry G: Diabetes mellitus and Alzheimer's disease: glycation as a biochemical link. Diabetologia 1996;39:247.

39 Luchsinger JA, Mayeux R: Cardiovascular risk factors and Alzheimer's disease. Curr Atheroscler Rep 2004;6:261-266.

40 van Harten B, et al: Cognitive impairment and MRI correlates in the elderly patients with type 2 diabetes mellitus. Age Ageing 2007;36:164-170

41 Beeri MS, et al: Insulin in combination with other diabetes medication is associated with less Alzheimer neuropathology. Neurology 2008:71:750-757

42 Friedman SM, et al: Hazards of hospitalization: residence prior to admission predicts outcomes. Gerontologist 2008;48:537-541.

43 Zimmerman S, et al: How good is assisted living? Findings and implications from an outcomes study. J Gerontol B Psychol Sci Soc Sci 2005;60:S195-S204.

44 Aud MA, Rantz MJ: Admissions to skilled nursing facilities from assisted living facilities. J Nurs Care Qual 2005;20:16-25.

45 American Diabetes Association: Standards of medical care in diabetes - 2008. Diabetes Care 2008;31(suppl 1):S12-S54.

$\checkmark 46$ Murthy SB, Jawaid A, Schulz PE: Diabetes mellitus and dementia: advocating an annual cognitive screening in patients with diabetes mellitus. J Am Geriatr Soc 2008;56:19761977. 